\title{
Editorial: The Chemical Ecology of Host and Mate Selection
}

\author{
Sebastien Lebreton ${ }^{1 *}$, Ahmed M. Saveer ${ }^{2}$, Richard A. Fandino ${ }^{3}$ and \\ William Benjamin Walker III ${ }^{4}$ \\ ${ }^{1}$ Chemical Ecology Department, Research Institute in Semiochemistry and Applied Ethology (IRSEA), Apt, France, \\ ${ }^{2}$ Department of Entomology and Plant Pathology, North Carolina State University, Raleigh, NC, United States, ${ }^{3}$ Department \\ of Ecology and Evolutionary Biology, Cornell University, Ithaca, NY, United States, ${ }^{4}$ Temperate Tree Fruit and Vegetable \\ Research Unit, United States Department of Agriculture - Agricultural Research Service (USDA-ARS), Wapato, WA, \\ United States
}

Keywords: semiochemicals, pheromones, sensory receptors, behavior, evolution, insects

Editorial on the Research Topic

The Chemical Ecology of Host and Mate Selection

\section{INTRODUCTION}

Chemical ecology studies the inter- and intra-specific chemical interactions shaped by the potential for an organism to seek out nutrients or a reproductive source efficiently. These processes are under strong selection pressure and are ultimately governed by the behavior of the animal toward physiologically relevant semiochemicals (Hansson and Stensmyr, 2011). Disentangling these behaviors have led to continuous advances in analytical chemistry, electrophysiology, neurogenetics, genomics, and integrative pest management strategies. With this Research Topic "The Chemical Ecology of Host and Mate Selection," we have sought to highlight the diversity of techniques used by chemical ecologists. In these reports, using different insect species, semiochemicals were functionally characterized both in the lab and in the field. The diversity of

\section{OPEN ACCESS}

Edited and reviewed by:

Maria L. Pappas,

Democritus University of

Thrace, Greece

*Correspondence:

Sebastien Lebreton

s.lebreton@group-irsea.com

Specialty section: This article was submitted to

Chemical Ecology,

a section of the journal

Frontiers in Ecology and Evolution

Received: 21 September 2021

Accepted: 08 October 2021

Published: 04 November 2021

Citation:

Lebreton S, Saveer AM, Fandino RA and Walker WB III (2021) Editorial: The

Chemical Ecology of Host and Mate Selection. Front. Ecol. Evol. 9:780540.

doi: 10.3389/fevo.2021.780540 chemosensory genes in understudied sensory tissues was examined, asking how this complexity and apparent redundancy of these genes may be key in determining selective behaviors that facilitate routes toward reproductive isolation.

\section{SELECTIVITY IN MATE ATTRACTION}

Mate attraction is often exemplified as a robust and highly sensitive physiological and behavioral response toward a conspecific mediated by one or two sex-specific pheromone compounds. The situation is, however, often more complex. Several species rely on the complex chemical signature formed by cuticular hydrocarbons (CHCs) for finding a mating partner (Blomquist and Bagneres, 2010). Three reports on parasitoid wasps exemplify the complex role of CHCs and other courtship specific compounds in inducing behaviors related to mate selection.

Würf et al. identify compounds that make up the female and male $\mathrm{CHC}$ blends in the Pteromalid wasp Urolepis rafepis. While the profiles of both sexes are qualitatively similar, they are quantitatively different, and only the female $\mathrm{CHC}$ extract elicits male courtship behavior. In a similar case of monomorphic CHC profiles in the Encyrtidid wasp, Tachinaephagus zealandicus, Jungwirth et al. found that male courtship behavior was only elicited with the female blend. Both studies highlight the importance of the context in which minor components are presented. Böttinger and Stökl present post-eclosion dispersal as a social behavior that would support reproductive fitness in four Leptopilina (Figitidae) species with varying pheromone volatility. The authors present a correlation between the volatility of the female pheromone and the male dispersal behavior from the natal patch. Species with pheromones of higher volatility disperse 
sooner compared to those that rely on contact pheromones. These results highlight the link between the evolution of pheromone volatiles and insect behaviors that might preclude the adaptation toward long-distance mating signals.

\section{SELECTIVITY IN HOST ATTRACTION}

An adequate host must provide key nutrients and shelter for an organism and its progeny. It is critical to quickly identify key semiochemicals that decisively inform direction and quality of the source (Janz, 2011). In this section we cover observations that describe the attraction of major agricultural pests toward their hosts. The knowledge acquired on these key semiochemicals involved in host selection may be applied to manipulate the behavior of pests by luring them into traps to protect cultivated crops (El-Shafie and Faleiro, 2017). Akotsen-Mensah et al. report on the attraction of Halyomorpha halys, a generalist pest of major crops toward single compounds and blends associated with their hosts. The authors demonstrate that the tri-trophic interaction with its parasitoid (Trissolcus japonica) is linked through common host volatiles. In the Coffee White Stem Borer (Xylotrechus quadripes), a major pest of coffee plants, Rajus et al. take a unique approach to exploring host attraction under the context of local agricultural conditions. In this work, promising compounds and blends are identified through rigorous physiological characterization coupled with analytical chemistry of two coffee host plants. Both attractive and deterrent semiochemicals were identified and tested in field traps. The authors demonstrate an efficient strategy for pest management and the importance of selectivity and environmental background in the delivery of attractants in lures. Lastly, Larson et al. also considered the importance of environmental context by describing lure efficiency in attracting the spotted-wing drosophila. This study dramatically exemplifies the importance of the environmental context in determining widely applicable and robust trapping mechanisms for monitoring pest populations. Overall, these reports demonstrate the importance in identifying key compounds that mark sensitivity toward the host but also account for the selectivity that is determined by more nuanced factors. A challenge arises in assessing the efficiency of these trapping systems not only through varying environmental factors but also under the impact of climate change.

\section{REFERENCES}

Auer, T. O., Khallaf, M. A., Silbering, A. F., Zappia, G., Ellis, K., ÁlvarezOcaña, R., et al. (2020). Olfactory receptor and circuit evolution promote host specialization. Nature 579, 402-408. doi: 10.1038/s41586-020-2 073-7

Blomquist, G. J., and Bagneres, A.-G. (2010). Insect Hydrocarbons: Biology, Biochemistry, and Chemical Ecology. Cambridge: Cambridge University Press. doi: 10.1017/CBO978051171 1909

Depetris-Chauvin, A., Galagovsky, D., Grosjean, Y., and Anderson, A. (2015). Chemicals and chemoreceptors : ecologically relevant signals driving behavior in Drosophila. Front. Ecol. Evol. 3:41. doi: 10.3389/fevo.2015.00041

\section{EVOLUTION OF HOST AND MATE SELECTION}

We finalize by exploring how host and mate selections are influenced and may evolve by previously unknown chemosensory pathways. Semiochemicals are detected by receptors and other proteins expressed in sensory neurons found throughout the body of an insect (Depetris-Chauvin et al., 2015; Vogt et al., 2021). Koutroumpa et al. sought to explore the chemosensory receptors expressed in neglected tissues in the moth Spodoptera littoralis, a model organism in the study of insect olfaction. Their work describes many previously unidentified chemosensory genes, some of which are sex biased in their expression. Receptors with a sexually dimorphic expression are likely to be involved in sex specific behaviors such as mating or oviposition. Therefore, in using a transcriptomic approach, Koutroumpa et al. open new avenues in understanding a possible role for the integration of volatile and contact semiochemicals in host and mate selection.

Finally, our topic offers an insightful review by Wang et al. that takes on the question of resource-mediated speciation through an original step-wise approach. Citing recent works exploring resource mediated speciation and metabolic adaptation (Karageorgi et al., 2019; Auer et al., 2020) the authors build an argument toward resource heterogeneity as a source of evolutionary avenues. Resource heterogeneity, rather the diversity of semiochemicals a species will encounter in its environment, may establish the necessary chemosensory machinery to support adaptive routes 0toward specific and non-competitive food sources. The authors also ask an important question: Is attraction and exploitation of novel resources genetically linked? And if so, is this linkage necessary or sufficient in determining resource-based assortative mating. Many points raised in this review directly reflect some of the questions and works we have compiled in this topic.

\section{AUTHOR CONTRIBUTIONS}

All authors listed have made a substantial, direct and intellectual contribution to the work, and approved it for publication.

El-Shafie, H. A. F., and Faleiro, J. R. (2017). "Semiochemicals and their potential use in pest management," in Biological Control of Pest and Vector Insects, ed V. D. C. Shields (Rijeka: InTech). doi: 10.5772/6 6463

Hansson, B. S., and Stensmyr, M. C. (2011). Evolution of insect olfaction. Neuron $\quad 72, \quad 698-711$. doi: 10.1016/j.neuron.2011.1 1.003

Janz, N. (2011). Ehrlich and Raven revisited: Mechanisms underlying codiversification of plants and enemies. Annu. Rev. Ecol. Evol. Syst. 42, 71-89. doi: 10.1146/annurev-ecolsys-102710-14 5024

Karageorgi, M., Groen, S. C., Sumbul, F., Pelaez, J. N., Verster, K. I., Aguilar, J. M., et al. (2019). Genome editing retraces the evolution of toxin resistance 
in the monarch butterfly. Nature 574, 409-412. doi: 10.1038/s41586-019-1 $610-8$

Vogt, R. G., Sparks, J. T., Fandino,. R. A., and Ashourian, K. T. (2021). "Reflections on antennal proteins: the evolution of pheromone binding proteins; diversity of pheromone degrading enzymes; and the distribution and behavioral roles of SNMPs," in Insect Pheromone Biochemistry and Molecular Biology, eds G. Blomquist and R. G. Vogt (London: Elsevier Academic Press). doi: 10.1016/b978-0-12-819628-1.00 021-3

Conflict of Interest: The authors declare that the research was conducted in the absence of any commercial or financial relationships that could be construed as a potential conflict of interest.
Publisher's Note: All claims expressed in this article are solely those of the authors and do not necessarily represent those of their affiliated organizations, or those of the publisher, the editors and the reviewers. Any product that may be evaluated in this article, or claim that may be made by its manufacturer, is not guaranteed or endorsed by the publisher.

Copyright (c) 2021 Lebreton, Saveer, Fandino and Walker. This is an open-access article distributed under the terms of the Creative Commons Attribution License (CC $B Y)$. The use, distribution or reproduction in other forums is permitted, provided the original author(s) and the copyright owner(s) are credited and that the original publication in this journal is cited, in accordance with accepted academic practice. No use, distribution or reproduction is permitted which does not comply with these terms. 